\title{
Inkjet Printed Wireless Smart Bandage
}

\author{
Muhammad Fahad Farooqui \\ Electrical Engineering Program \\ King Abdullah University of Science and Technology \\ (KAUST) \\ Thuwal, Saudi Arabia
}

\author{
Atif Shamim \\ Electrical Engineering Program \\ King Abdullah University of Science and Technology \\ (KAUST) \\ Thuwal, Saudi Arabia
}

\begin{abstract}
Chronic wounds affect millions of patients around the world and requires a major portion of health care budget for treatment. In this article, we present an unprecedented low cost continuous wireless monitoring system, realized through inkjet printing on a standard bandage strip, which can send early warnings as well as record long term wound progression data. The smart bandage can communicate upto a distance of $60 \mathrm{~m}$ when worn on the body.
\end{abstract}

Keywords-Smart badnage; inkjet printing; chronic wounds

\section{INTRODUCTION}

Increase in population and rising cost of healthcare services have created a growing demand to monitor a patient's health in personal environment outside of a hospital. For this purpose, wearable sensors are being developed to monitor various physiological parameters of the human body. Chronic wounds affect more than 9 million people in the United States and their treatment costs exceed US\$ 29 billion [1]. Diagnosis and the treatment of chronic wounds are quite complex and pose a major challenge to the health care staff. One of the major causes of disruption of healing process in wounds is bacterial infection. An infected wound show frequent and irregular bleeding [2]. Changes in $\mathrm{pH}$ values have also been related to the presence of infection [3]. Moreover pressure ulcer can develop if a part of the body is under sustained pressure for a longer period of time [4].

Few attempts to monitor parameters related to the wound healing process have been made [5,6]. However, there is currently no commercially available solution to continuously monitor the wound healing process and patients rely on medical staff for physical inspection of the wound, which requires repeated trips to clinics or prolonged hospitalization.

Here, we present for the first time, a complete wireless, realtime wound monitoring system using a simple bandage strip that can be worn in daily life. The system, termed as smart bandage, comprises low cost inkjet printed sensors to monitor multiple parameters like bleeding, $\mathrm{pH}$ levels and external pressure on the wound site. The sensors have been directly printed on a commercial bandage strip whereas the wireless electronics is on a flexible tape, thus making the sensors disposable and electronics reusable. The bandage can issue early warnings to the patients regarding any abnormality in the

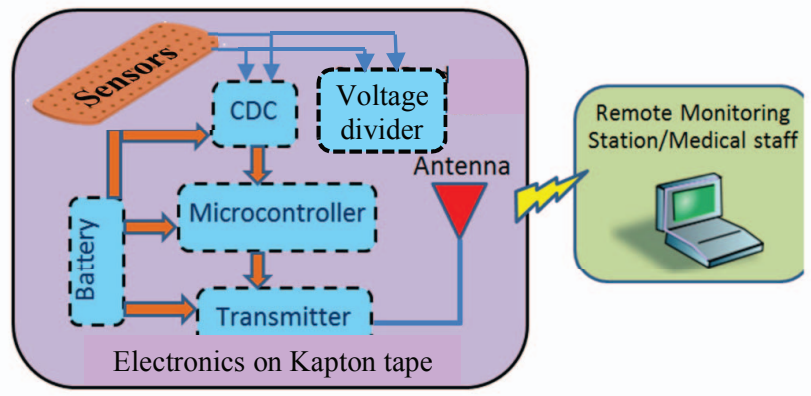

Fig. 1. System Design

healing process, as well as wirelessly send recordings of wound progression data to the remote medical staff. In this way, health care providers would have access to the history of wound progression that could be vital in the diagnosis and treatment process.

\section{SYSTEM DESIGN AND OPERATION}

The system level design is depicted in Fig. 1, which shows the disposable sensors on a disposable bandage whereas the electronics are integrated on a flexible kapton tape which can be detached and reused multiple times. Two types of sensing mechanisms are used. A capacitive sensor detects bleeding as well as pressure levels on the wound. A resistive sensor detects the $\mathrm{pH}$ levels on the wound. A capacitance to digital converter (CDC, On Semiconductor ${ }^{\circledR}$ LC717A00AJ) continuously compares the sensor capacitance to a reference capacitance and outputs a logic high when the sensor capacitance becomes greater than the reference capacitance as a result of bleeding or external pressure. Similarly, the output from the resistive sensor is converted into voltage through a voltage divider. A microcontroller (Texas Instruments CC2530) continuously monitors the voltage levels from the CDC and voltage divider and as soon as it detects a signal, it activates the transmitter as well as the LED. The wireless communication is done through a printed loop antenna that is integrated with the circuit. The double-sided circuit layout as well as the antenna has been realized on kapton tape using low cost inkjet printing technology. The smart bandage can wirelessly communicate to remote health care providers via 


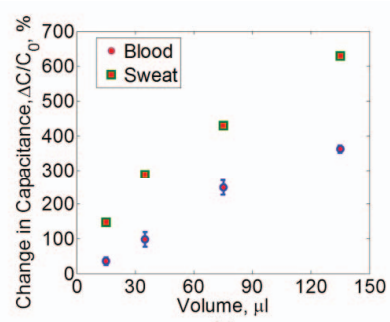

(a)

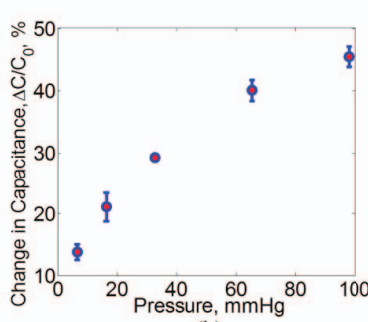

(b)

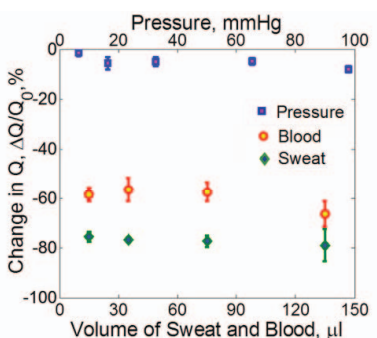

(c)

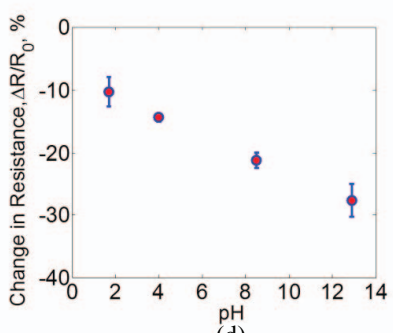

(d)

Fig. 2. Sensor measurements. (a) Bleeding (b) Pressure (c) Quality factor of capacitive sensor. (d) pH

smart phone using either the mobile network or the internet.

A capacitive sensor is formed by placing two electrodes on either sides of the bandage strip. The two electrodes are therefore separated by the thickness of the bandage strip. The kapton PCB tape carries the top electrode. In the case that the wound starts to bleed, blood from the wound will penetrate into the bandage and change the dielectric constant of the bandage resulting in a change of capacitance. The external pressure is sensed due to the change in thickness of the bandage. When pressure is applied to the bandage, the two electrodes of the capacitor are pressed together which causes a change in capacitance. The $\mathrm{pH}$ levels on the wound site are detected by changes in resistance of the carbon based bottom electrode. When exposed to a solution containing hydronium ions $\left(\mathrm{H}_{3} \mathrm{O}^{+}\right)$in the case of an acid or hydroxide ions $\left(\mathrm{OH}^{-}\right)$in the case of a base, the carbon reacts with these ions which results in a change of its conductivity.

\section{EXPERIMENTAL RESULTS}

\section{A. Bleeding, Pressure and $\mathrm{pH}$ Sensing}

The capacitor sensor placed across the bandage senses bleeding. In order to mimic blood, a mixture of water and ethanol is prepared that has a similar dielectric constant as that of blood. Different volumes of this mixture are then dropped on the bandage and the capacitance is measured across the two sensing electrodes. As can be seen in Fig. 2(a), a few micro liters of this fluid change the capacitance significantly. To measure external pressure using the capacitive sensor, different values of mass are put on the bandage in an area of $1 \mathrm{~cm}^{2}$. The results are shown in Fig. 2(b). A pressure of around $20 \mathrm{mmHg}$ produces a capacitance change of around 10\%. Quality factor measurements, Fig. 2(c), show that bleeding can be clearly distinguished from pressure even though both are measured by the same capacitive sensor.

For $\mathrm{pH}$ measurements, solutions of fixed $\mathrm{pH}$ values are prepared. Line traces are made using the carbon ink that have an initial resistance of around $2.5 \mathrm{k} \Omega$. The traces are exposed to $5 \mu \mathrm{l}$ of each of the $\mathrm{pH}$ solutions. The resistance changes immediately after the exposure and takes some time to stabilize due to the absorption of the solution. The measured resistance is shown in Fig. 2(d). It can be seen that the resistance decreases with increasing $\mathrm{pH}$ values.

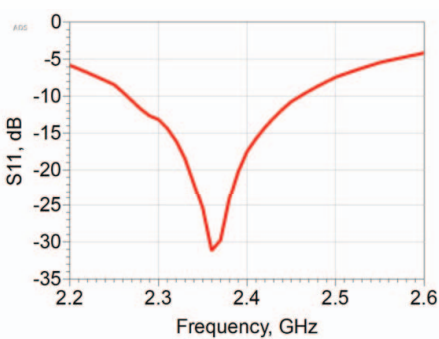

(a)

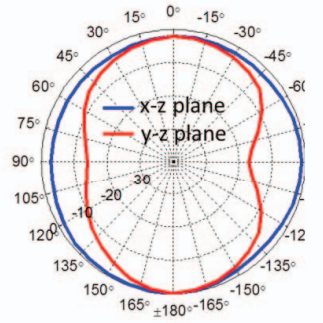

(b)
Fig. 3. Antenna measurements

\section{B. Wireless Tests}

An active radiation pattern measurement of the antenna has been measured. The active pattern is shown in Fig. 3 along with the reflection coefficient of the antenna. As can be seen the active pattern is omnidirectional as expected from a loop antenna. Also the antenna is impedance matched at the operating frequency of $2.4 \mathrm{GHz}$. Field tests have been carried out to measure the communication range of the bandage. The range has been measured with the bandage on a human body as well as in air. A range of around $60 \mathrm{~m}$ is measured when the bandage is placed on a body.

\section{REFERENCES}

[1] Doebbeling, B. N. The validity of the clinical signs and symptoms used to identify localized chronic infection. Wound Repair and Regeneration 9, 178 (2001)

[2] Hampton, S. Understanding overgranulation in tissue viability practice. British Journal of Community Nursing 12, 9 (2007)

[3] Schreml, S., Szeimies, R-M, Karrer, S., Heinlin, J., Landthaler, M. $\&$ Babilas, P. The impact of the $\mathrm{pH}$ value on skin integrity and cutaneous wound healing. J. European Academy Dermatology Venereology 24, 373 (2010)

[4] Dharmarajan, T. S. \& Ugalino, J. T. Pressure ulcers: Clinical features and management. Hospital Physician 38, 64 (2002)

[5] Li, Z. et al. Non-invasive transdermal two-dimensional mapping of cutaneous oxygenation with a rapid drying liquid bandage. Biomedical Optics Express 5, 3748 (2014)

[6] Guinovart, T., Ramirez, G. V-., Windmiller, J. R., Andrade, F. J. \& Wang, J. Bandage-based wearable potentiometric sensor for monitoring wound pH. Electroanalysis 26, 1345 (2014). 